\title{
THE NOVEMBER MEETING IN RALEIGH
}

The four hundred eighty-fifth meeting of the American Mathematical Society was held at North Carolina State College of Agriculture and Engineering of the University of North Carolina, Raleigh, N. C., on Friday and Saturday, November 28-29, 1952. The total attendance was 142, including the following 94 members of the Society:

Milton Abramowitz, R. L. Anderson, N. C. Ankeny, A. T. Brauer, E. T. Browne, R. C. Bullock, E. A. Cameron, Leonard Carlitz, Virginia Carlock, C. L. Carroll, J. W. Cell, J. M. Clarkson, Haskell Cohen, J. C. Currie, D. F. Dawson, B. V. Dean, W. J. Dixon, F. G. Dressel, B. M. Drucker, W. L. Duren, Jr., S. E. Dyer, J. C. Eaves, W. W. Elliott, D. O. Ellis, M. E. Estill, H. A. Fisher, J. W. Gaddum, J. R. Garrett, I. C. Gentry, J. J. Gergen, Seymour Ginsburg, Wallace Givens, Herbert Goertzel, H. H. Goldstine, D. B. Goodner, E. E. Grace, H. C. Griffith, E. H. Hadlock, R. R. Hare, Jr., O. G. Harrold, E. A. Hedberg, P. S. Herwitz, A. T. Hind, O. H. Hoke, Harold Hotelling, A. S. Householder, G. B. Huff, Ernest Ikenberry, J. R. Isbell, F. B. Jones, John Jones, Jr., R. J. Koch, F. W. Kokomoor, H. T. LaBorde, G. B. Lang, J. W. Lasley, Jr., T. H. Lee, R. J. Levit, C. F. Lewis, P. E. Lewis, W. R. Longley, E. L. Mackie, J. S. MacNerney, W. R. Mann, G. W. Medlin, E. P. Miles, Jr., H. C. Miller, Benjamin Ernest Mitchell, J. C. Morelock, C. G. Mumford, H. M. Nahikian, J. D. Novak, F. R. Olson, H. V. Park, W. V. Parker, B. J. Pettis, T. J. Pignani, J. H. Roberts, W. A. Rutledge, A. L. Shields, J. R. Shoenfield, C. B. Smith, W. S. Snyder, W. L. Strother, P. M. Swingle, Olga Taussky, John Todd, R. Z. Vause, J. H. Wahab, M. J. Walsh, H. C. Wang, W. M. Whyburn, L. S. Winton, G. N. Wollan.

By invitation of the Committee to Select Hour Speakers for Southeastern Sectional Meetings, Professor Wallace Givens of the University of Tennessee and Dr. H. H. Goldstine of The Institute for Advanced Study addressed the Society. Dr. Goldstine's address, entitled Some remarks on numerical analysis, was delivered at 8:00 P.M. on Friday, with Dr. A. S. Householder presiding. The address by Professor Givens, entitled Polarities and their signature in von Neumann's continuous geometry, was given at 10:00 A.M. on Saturday, with Professor Gerald Huff presiding.

Four sessions for the presentation of contributed papers were held on Friday afternoon and Saturday morning. Presiding officers for these sessions were Professors J. H. Roberts, F. W. Kokomoor, W. V. Parker, and B. J. Pettis.

All sessions were held in Room 242 of the Riddick Laboratories Building on the campus of the North Carolina State College. Lounges and conference rooms in the building were also used. A resolution expressing the appreciation of the Society to its hosts for the meeting was offered by Professor W. L. Duren and unanimously adopted. 
Abstracts of the papers presented follow below. Abstracts whose numbers are followed by the letter " $t$ " were presented by title. Paper number 51 was read by Professor Rutledge. Professor J. W. Ellis was introduced by Dr. Goodner, Mr. McAuley by Professor Jones, Dr. Jaeger by Professor Cairns, Dr. Gordon and Mr. Capel by Professor Wallace, Mr. Pellicciaro and Dr. Shields by Professor Whyburn.

\section{Algebra ANd Theory of Numbers}

\section{A. T. Brauer: On the distribution of Jacobian symbols.}

Let $p$ be a prime and $e_{\nu}=0$ or $\pm 1(\nu=1,2)$. N. Aladow (Mat. Sbornik vol. 18 (1896) pp. 61-75) determined the number of integers $a$ in a complete system of residues $(\bmod p)$ for which the Legendre symbols $(a / p)=e_{1}$ and $(a+1 / p)=e_{2}$ for each given $e_{1}$ and $e_{2}$. Other proofs for these results were given by E. Jacobsthal (Anwendung einer Formel aus der Theorie der quadratischen Reste, Dissertation, Berlin (w. Fr. Kästner, Göttingen, 1906)) and by O. Perron (Math. Zeit. vol. 56 (1952) pp. 122-130). In this paper the corresponding problem for Jacobian symbols for odd squarefree moduli is solved. These results will be used in another paper for the construction of Hadamard determinants of certain orders. (Received November 28, 1952.)

\section{8t. Leonard Carlitz: $A$ note on modular invariants.}

Consider a system of forms $f_{1}, \cdots, f_{r}$, with coefficients $\in G F(q)$, which are subjected to the transformation of the full linear group over $G F(q)$; thus the number of classes is some finite number $k$. Let the invariants of the system take on values in an arbitrary field $\Phi$. We show that if $\Phi$ contains at least $k$ distinct numbers, then there exists an invariant $J$ such that every invariant can be exhibited as a polynomial in $J$ of degree $\leqq k-1$ and with coefficients in $\Phi$. More generally if $\Phi=G F\left(q_{1}\right)$ and $q_{1}^{i-1}$ $<k \leqq q_{1}$, then there exist $s$ invariants $J_{1}, \cdots, J_{s}$ such that every invariant is a polynomial in the $J$ 's. Moreover the number $s$ cannot be diminished. (Received October $6,1952$.

39t. Leonard Carlitz: A reciprocity formula for weighted quadratic partitions.

This note is concerned with a reciprocity theorem for the sum $S(\alpha, \lambda, Q)$ $=\sum_{Q(\xi)-\alpha} e\left(2 \lambda_{1} \xi_{1}+\cdots+2 \lambda_{r} \xi_{r}\right)$, where $\alpha, \lambda_{i}, \xi_{i} \in G F(q)$ and $Q$ is a quadratic form with coefficients in $G F(q)$. Some extensions and a rational analogue are also discussed. (Received October 6, 1952.)

40. Leonard Carlitz: Invariant theory of systems of equations in a finite field.

The results of the author's paper: Invariant theory of equations in a finite field are extended to the general case. (Received October 6, 1952.)

41. B. V. Dean: Regular isotopes of a near ring.

A near ring $N$ is a system having two laws of operation, addition and multiplication, where (1) addition is single-valued and has a zero element, (2) multiplication is single-valued and associative, (3) $a o=o=o a, a$ in $N$, and (4) multiplication is left 
distributive over addition. $e$ is left (right) regular if $e a=e b(a e=b e)$ implies $a=b$. The totality of functions on a system satisfying (1), which preserve zero, is a near ring. $(A, B, C)$ is an isotopy of $N$ onto the near ring $M$, and $M$ is an isotope of $N$, if $A, B$, and $C$ are three additive isomorphisms of $N$ onto $M$ and $(a b)^{C}=a^{A} b^{B}, a, b$ in $N$. An isotopy is a regular isotopy if $N$ has a left regular element $e$ and a right regular element $f$ with $(e N)^{A}=e^{A} M$ or $(N f)^{B}=M f^{B}$. Regular isotopic near rings are isomorphic under a mapping $D$ and if $N$ has a multiplicative identity the isotopy is a multiplication of the isomorphism $A C^{-1} B=D=B C^{-1} A$. Examples show that isotopic subrings of a commutative field may not be isomorphic and for regular isotopes we may have $A C^{-1} B \neq D \neq B C^{-1} A$. (Received October 7, 1952.)

\section{2t. Trevor Evans and B. H. Neumann: On varieties of groupoids} and loops.

An infinite irredundant system of groupoid laws is described; it follows that the set of varieties (in the sense of P. Hall) of groupoids has the cardinal $c$ of the continuum. Also an infinite irredundant system of loop laws is exhibited; loops are here equationally defined in terms of three binary operations: multiplication, right division, and left division (cf. Trevor Evans, J. London Math. Soc. vol. 24 (1949) pp. 254-260). It follows that the set of varieties of loops has cardinal c, and so has the set of varieties of quasigroups. The construction of both infinite irredundant systems of laws utilizes an infinite irredundant system of group relations taken from B. H. Neumann, J. London Math. Soc. vol. 12 (1937) pp. 120-127. It is further shown that power associativity of loops or quasigroups or groupoids is necessarily an infinite set of laws, though no irredundant system of laws equivalent to power associativity in loops is known, and the existence of such a system is highly doubtful. (Received October 20, 1952.)

43. E. H. Hadlock: Primitive ternary indefinite quadratic genera of more than one class.

Genera, of properly primitive forms with improperly primitive reciprocals, containing at least two classes are shown to exist. The invariants $\Omega$ and $\Delta$ associated with the forms are defined by $\Omega=\Omega^{\prime} \Omega^{\prime \prime}, \Delta=\Delta^{\prime} \Delta^{\prime \prime}=\Delta^{\prime}, \Omega^{\prime}=\Omega_{1}^{2} P_{\Omega \Delta}, \Delta^{\prime}=-\Delta_{1}^{2} P_{\Omega \Delta}$ where $\Omega^{\prime \prime}$ and $\Delta^{\prime \prime}$ are the largest powers of 2 dividing $\Omega$ and $\Delta$ respectively, and $\Omega_{1}^{2}$ and $\Delta_{1}^{2}$ are the largest squares dividing $\Omega^{\prime}$ and $\Delta^{\prime}$ respectively. $1<P_{\Omega \Delta} \equiv 1(\bmod 8)$. Also $\Omega^{\prime \prime} \geqq 64$. Each of the characters associated with the forms has the value one. (Received October 14, 1952.)

\section{4t. A. J. Hoffman and Olga Taussky: A characterization of normal matrices.}

One of the definitions for a matrix $A$ to be normal is that $A A^{*}=A^{*} A$ where $A^{*}$ is the transposed and conjugate matrix of $A$. Commutativity of two matrices $A, B$ implies that every polynomial $p(A, B)$ has as characteristic roots $p\left(\alpha_{i}, \beta_{i}\right)$ where $\alpha_{i}$ are the characteristic roots of $A$ and $\beta_{i}$ of $B$ in a special ordering. While this property is weaker than commutativity for general matrices $A, B$, it implies commutativity if $B=A^{*}$. It can even be shown that either of the two following facts implies the normality of $A$ : (1) $A+A^{*}$ has as characteristic roots $\alpha_{i}+\bar{\alpha}_{k}$ for some ordering. (2) $A A^{*}$ has as characteristic roots $\alpha_{i} \bar{\alpha}_{k}$ for some ordering. (Received September 22, 1952.)

\footnotetext{
45. G. B. Huff: Matrices such that $A^{t}$ is a polynomial in $t$ and principal idempotent elements.
} 
Let $D$ be an integral domain (with identity) and of characteristic $p=0$, and $D_{m}$ be the ring of $m$ by $m$ matrices with elements in $D$. For $E_{\theta}, A \in D_{m}, E_{0}$ is said to be an identity for $A$ if $E_{g}^{2}=E_{g}$ and $E_{g} A=A E_{g}=A$. A matrix $A$ is said to be $E_{g} F(X)$ if and only if $E_{g}$ is an identity for $A$ and there exists a matric polynomial $F(X) \in D_{m}[X]$ such that $F(0)=E_{g}$ and $F(t)=A^{t}$ for each natural number $t$. If $n$ is a natural number and $E_{g}$ is an identity for $A$, then the principal result states that $A$ is $E_{g} F(X)$ with $F(X)$ of degree $n$ if and only if $A-E_{o}$ is nilpotent of index $n+1$. When $D$ is the field of complex numbers, this is connected with the notion of the principal nilpotent and idempotent elements [Wedderburn, Colloquium lectures, pp. 28-30] of $A$ in the following way. If $E_{j}$ is the principal idempotent element associated with a nonzero characteristic root $x_{j}$ of $A$, then $x_{j}^{-1} A E_{j}$ is $E_{j} F(X)$ where $F(X)$ is of degree one less than the multiplicity of $x_{i}$ as a root of the minimum function of $A$. The clarity with which some classical and some new results come out of this connection suggests that the notion of $E_{g} F(X)$ matrices belongs at the beginning of matrix theory. (Received October $9,1952$.

\section{Arno Jaeger: Linear differential equations in fields of prime number characteristic.}

An iterative differentiation $D$ in a field $F$ of characteristic $p \neq 0$ in the sense of $F . K$. Schmidt (J. Reine Angew. Math. vol. 190 (1952) p. 4) with the property $D a \neq 0$ for at least one $a \in F$ is used to define modified formal differential equations in $F$. A generalization of the basis theorem for these differentiations is the main tool to calculate the solutions of the differential equations by solving algebraic equations only. Hence solutions of a differential equation can be found already in algebraic extensions of $F$ provided that solutions exist at all (for instance $D y=a^{p-1}$ has no solution if $D a=1$ holds). The ring of all differential operators $\sum_{r-0}^{n} a_{r} D^{r}$ differs from its classical analogon by the existence of zero divisors caused by the iteration rule $D^{i} D^{i}=C_{i+j, i} D^{i+j}$. Thus new methods for finding the inverse of a given differential operator have to be adopted. The solutions of all linear differential equations with constant coefficients $\sum_{r=0}^{n} a_{r} D^{r} y=v$ can be given explicitly; if $a_{0} \neq 0$ there exists a unique solution. For arbitrary linear differential equations the number of independent parameters entering the general solution may be greater or less than its formal order, but an upper bound can be found. (Received October 14, 1952.)

\section{T. H. Lee: Matrices with generalized quaternions as elements.}

Let $\alpha$ and $\beta$ be square-free rational integers and $r_{0}, r_{1}, r_{2}$, and $r_{3}$ real numbers. We define $i$ and $j$ by the equations $i^{2}=-\alpha, j^{2}=-\beta$, and $i j=-j i$. The elements $r=r_{0}+r_{1} i$ $+r_{2} j+r_{3} i j$ form a real generalized quaternion algebra $Q^{\prime}(\alpha, \beta)=Q^{\prime}$. If both $\alpha$ and $\beta$ are positive, $Q^{\prime}$ is isomorphic to the ordinary real quaternions. But $Q^{\prime}$ is not even a division algebra if either $\alpha$ or $\beta$ is less than zero. Louise A. Wolf (1935), H. C. Lee (1949), and J. L. Brenner (1951) have studied certain properties of matrices whose elements are ordinary real quaternions. In this paper, matrices $A$ whose elements are generalized quaternions are studied. An element $\lambda$ of $Q^{\prime}$ is called a right-hand characteristic root of $A$ if a nonzero vector $x$ with components in $Q^{\prime}$ exists such that $A x=x \lambda$. It is proved here that such a root exists if at least one of $\alpha$ and $\beta$ is positive. It remains unsolved whether characteristic roots always exist if $\alpha$ and $\beta$ are negative. But similar matrices either have no roots or the same roots $\lambda_{k}$. Transforms $\rho \lambda_{k} \rho^{-1}$ of $\lambda_{k}$ are themselves characteristic roots for each $\rho$ in $Q^{\prime}$ with a nonvanishing norm. Moreover, if $A$ is triangular, a characteristic root $\lambda_{k}$ always exists, and for each characteristic root $\lambda_{k}$ a nonzero element $x$ of $Q^{\prime}$ exists such that $a_{s s} x=x \lambda_{k}$ where $a_{s s}$ is an 
element of the main diagonal of $A$. It is shown that one of the $a_{s s}$ is always a characteristic root, but not necessarily all of them are. (Received November 28, 1952.)

\section{R. J. Levit: Division rings in terms of a single operation.}

It was shown by N. Wiener [Trans. Amer. Math. Soc. vol. 21 (1920) pp. 237-246] that a field can be characterized as a system with only one undefined operation $a \nabla b$, which can be expressed in terms of the usual field operations as $1-a / b$. Another characterization in terms of the single class-closing operation $a \Delta b=a(1-b)$ was given by the author [Trans. Amer. Math. Soc. fol. 57 (1945) pp. 426-440]. In the present paper this work is extended to the non-commutative case. Sets of postulates for a division ring are given in terms of each of the operations $a \triangle b$ and $a \nabla b=1-b^{-1} a$. (Received October 14, 1952.)

49. G. W. Medlin: A note on a theorem of Parker.

Recently W. V. Parker [Proceedings of the American Mathematical Society vol. 1 (1950)] proved the following theorem. Let $A$ be an $n \times m$ matrix of rank $r$ such that $A C A=k A$ ( $k$ a scalar). If $B$ is an $m \times n$ matrix, the characteristic equation of $A B$ is $x^{n-r} \phi(x)=0$ and that of $A(B+C)$ is $x^{n-r} \phi(x-k)=0$. In the same manner that $\mathrm{W}$. $\mathrm{T}$. Reid [Proceedings of the American Mathematical Society vol. 1 (1950)] generalized an earlier theorem of Parker [Bull. Amer. Math. Soc. vol. 55 (1949)], this result will be generalized by proving the following theorem. Let $A$ be an $n \times m$ matrix of rank $r$ and $D$ an $n \times n$ matrix such that $D A=k A$ ( $k$ a scalar). If $B$ is an arbitrary $m \times n$ matrix, then the characteristic equation of $A B$ is $x^{n-r} \phi(x)=0$ and that of $A B+D$ is $g(x) \phi(x-k)=0$, where $g(x)$ is a polynomial of degree $n-r$. (Received November 28, 1952.)

50. W. V. Parker: Characteristic roots of a set of matrices.

A square matrix $A$ may be uniquely written as $A=H+i K$ where $H$ and $K$ are Hermitian matrices. The characteristic roots of $H$ and $K$ determine a rectangular grid in the complex plane. All characteristic roots of $A$ are within or on this grid. All matrices $B$ given by $B=U H U^{*}+i V K V^{*}$, where $U$ and $V$ are unitary matrices, have the same associated grid. It is the purpose of this paper to examine the distribution of the characteristic roots of these matrices as $U$ and $V$ range over the set of all unitary matrices. Two special cases are considered. (Received October 15, 1952.)

51. W. V. Parker and W. A. Rutledge: Equivalence of matrices over a polynomial domain.

Let $a=\left(a_{1}, a_{2}, \cdots, a_{n}\right)$ be a vector over a field $F$ and $a(x)=a_{1}+a_{2} x+\cdots$ $+a_{n} x^{n-1}$. The vectors $e_{i}$ are defined by $e_{i}(x)=x^{i-1}$. A matrix whose rows are $e_{2}, e_{3}$, $\cdots, e_{n}, a$ is the companion matrix of $f(x)=x^{n}-a(x)$, denoted by $C(f)$. The matrix whose last row is the vector $a$, and other rows zeros, is the associate of $a(x)$, denoted by $A(a)$. Let $M=\left(M_{i j}\right)$ be a $k \times k$ block matrix, with $M_{i i}$ the companion matrix $C\left(f_{i}\right)$, and $M_{i j}, i \neq j$, the associate matrix $A\left(f_{i j}\right)$. It is shown that $M-x I$ is equivalent to diag. $\left\{I_{n-k}, M(x)\right\}$ where $M(x)$ is the $k \times k$ matrix $\left(m_{i j}\right)$ with $m_{i i}=f_{i}(x)$ and $m_{i j}=f_{i j}(x), i \neq j$. Several known results are corollaries (e.g. Theorem I in M.F. Smiley, The rational canonical form of a matrix, Amer. Math. Monthly vol. 56 (1949) p. 542). A simple proof using matrix theory is given of a theorem of Flanders $(H$. Flanders, Elementary divisors of $A B$ and $B A$, Proceedings of the American Mathematical Society vol. 2 (1951) p. 872). (Received October 15, 1952.) 
52t. W. E. Roth: On the characteristic polynomial of the product of two matrices.

The following theorem is proved: If $A$ and $B$ are $n \times n$ matrices with elements in the field $F$, whose characteristic polynomials are $a_{0}\left(x^{2}\right)-x a_{1}\left(x^{2}\right)$ and $b_{0}\left(x^{2}\right)-x b_{1}\left(x^{2}\right)$ respectively, where $a_{0}\left(x^{2}\right), a_{1}\left(x^{2}\right), b_{0}\left(x^{2}\right)$, and $b_{1}\left(x^{2}\right)$ are polynomials in $x^{2}$ in $F[x]$, the polynomial domain of $F$; and if the rank of $A-B$ is less than or equal to unity; then the characteristic polynomial of their product, $A B$, is $a_{0}(x) b_{0}(x)-x a_{1}(x) b_{1}(x)$. (Received September 23, 1952.)

\title{
53t. Olga Taussky: Generalized commutators of matrices.
}

It was proved by Shoda that any unimodular $n \times n$ matrix can be expressed as the commutator of two $n \times n$ matrices. A more general result is: if $X, Y$ are any two $n \times n$ matrices with equal nonvanishing determinants, then $n \times n$ matrices $C, D$ can be found such that $Y=C^{-1} D^{-1} X C D$. Applications of this fact are discussed. (Received September 22, 1952.)

\section{J. H.Wahab: New cases of irreducibility for Legendre polynomials. II.}

Let $P_{n}(x)$ be the Legendre polynomial of degree $n$. Let $p$ be any prime. If (1) $n=(p-1)\left(p^{k_{1}}+p^{k_{2}}+\cdots+p^{k_{i}}\right)$, where $k_{i} \neq k_{r}$ for $i \neq r$, and if $P_{n}(x)$ be reducible in the field of rational numbers, then the degree of each factor can be similarly expressed in terms of $p$ and each power of $p$ occurring in (1) will occur once and only once among the representations of the degrees of the factors. This necessary condition for reducibility if $p=2$ was proved in the author's first paper (Duke Math. J. vol. 19 (1952) pp. 165-176) and was used to obtain certain irreducible $P_{n}(x)$. A proof for arbitrary $p$ is given here and the result is applied to prove additional irreducible cases of $P_{n}(x)$. (Received October 15, 1952:)

\begin{abstract}
AnAlysis
55. Seymour Ginsburg: Some results on fixed points of simply ordered sets.

Let $A$ be a simply ordered set. The element $p$ of $A$ is called a fixed point of $A$ if $f(p)=p$ for each similarity transformation $f$ of $A$ into $A$. Theorem: Let $p$ be a fixed point of $A, q$ a fixed point of $B$, and let $A \times B$ be ordered by first differences. Then (a) either $p$ or $q$ is a fixed point of the ordered sum $A+B$; and (b) there exists a fixed point $(r, q)$ of $A \times B$, where $r$ is some fixed point of $A$ for which both of the sets, $\{x \mid r \leqq x \leqq p, x \in A\}$ and $\{x \mid p \leqq x \leqq r, x \in A\}$, are finite. (Received October 14, 1952.)
\end{abstract}

\section{Ernest Ikenberry: $A$ note on the Liouville equation $D f=0$.}

By decomposing the distribution function in phase space into its even and odd parts with respect to the momentum coordinates, so that $f=f_{0}+f_{0}$, and applying the operator $D$ (J. of Chem. Phys. vol. 19 (1951) pp. 467-470; vol. 20 (1952) pp. 568-570) to $f_{e}$ and $f_{0}$ separately, we find that, in the stationary case in which $\partial f / \partial t=0, D f_{e}$ is an odd function and $D f_{0}$ is an even function of the momentum coordinates, unless it is assumed that the intermolecular forces depend on the momenta. Hence we have not only $D f=0$, but also $D f_{0}=0$ and $D f_{0}=0$, in the stationary case; the distribution function is a superposition of two functions between which the Liouville equation gives no relation. In this case, Gibbs' principle of "conservation of density-in-phase" is 
inadequate for obtaining a relation between the heat current and the temperature gradient, or between the components of the strain tensor and the velocity gradients. To obtain such relations by application of Gibbs' principle it is necessary to consider the non-stationary case, or to assume that the force components $R_{i}$ depend on the momentum components $p_{i}$. (Received October 15, 1952.)

\section{7t. W. H. Ingram: An integral related to the Pollard-Moore-Stieltjes integral.}

Let $f$ be bounded and with simple discontinuities only, $g$ be in the class $B$ of functions of bounded variation, $2 \bar{f}(x) \equiv f(x+)+f(x)$, then $(M) \int_{a}^{b} f d g \equiv \lim _{\sigma} \sum_{i / \sigma} \bar{f}\left(x_{i}\right)$ $\left[g\left(x_{i+1}\right)-g\left(x_{i}\right)\right]$, with $\lim _{\sigma}$ the Smith-Moore limit, exists. The $M$-integral has an ordinary integration by parts formula for functions continuous on the left; it is the mean of the $N$-integral defined in Bull. Amer. Math. Soc. Abstract 55-7-350 and the $L C S \sigma$-integral of Price (Bull. Amer. Math. Soc. vol. 49 (1943) p. 627). Let $f \equiv(\bar{f}(x), \bar{f}(x+)), d \xi \equiv\left(j g, g^{\prime}(x+) d x\right), d x>0, j g \equiv g(x+)-g(x)$, then the lower (inner) vector product being understood, $(M) \int_{a}^{b} f d g=\lim _{\sigma} \sum_{i / \sigma} f\left(x_{i}\right) d_{\mathcal{g}}\left(x_{i}\right), d x=\Delta_{i} x$, for $g$ in that subclass of $\mathscr{B}$ such that $g^{\prime}(x+)$ exists and is bounded (i.e., is in $\mathfrak{B}^{\prime}$ ) and moreover such that the difference quotient $(g(x+\delta)-g(x+)) / \delta, \delta>0$, converges uniformly on $[a b]$ (i.e., is in $\mathfrak{B}^{*}$ ), also, $d \int_{a}^{x} f d g=f d \delta, a \leqq x \leqq b$, almost everywhere, $g$ in $\mathfrak{B}$, or everywhere, $g$ in $\mathfrak{B}^{\prime}$. The $M$-integral has effective application to the problem $d y=d H(x, \mu) y$, $L y(a)+R y(b)=0, H$ in $\mathfrak{B}^{*}$ and continuous on the left. (Received November 22, 1952.)

\section{J. S. MacNerney: Essential adjoints and balanced transforma-} tions.

The space $S$ is assumed to be linear and complete, with inner product $(x, y)$ notation is consistent with J. von Neumann, Functional operators (vol. II), Annals of Mathematics Studies, no. 22, Princeton, 1950; "transformation" means single-valued operator. The essential adjoint, $T^{e}$, of an operator $T$ is the operator with graph consisting of all ordered pairs of the form $\left\langle P_{B} y, P_{A} y^{\prime}\right\rangle$ where $\left\langle y, y^{\prime}\right\rangle$ belongs to the graph of $T^{*}, A=[D(T)]$, and $B=[R(T)]$; balanced operators are those operators $T$ such that $T^{\circ e}=T$. It is proved that (1) if $T$ is an operator, then $T^{\circ}$ is a closed linear reversible transformation, and (2) every closed linear reversible transformation is balanced. (Received October 14, 1952.)

\section{E. P. Miles: A note on harmonic functions generated by analytic} functions of a hypervariable.

In a preliminary report [Bull. Amer. Math. Soc. Abstract 58-1-61] the author considered a class of 3-dimensional harmonic functions generated by analytic functions of a certain hypervariable. A closely related class of harmonic functions is generated by analytic functions of the hypervariable $t=x+w y+w^{2} z$ where $w^{3}=1$. For analytic $f(t)=U(x, y, z)+w V(x, y, z)+w^{2} W(x, y, z)$ we have $V^{2} U=V^{2} V=V^{2} W$; thus the function $(w-1) f(t)$ has harmonic components $W-U, U-V$, and $V-W$. Let $H(x, y, z)$ be one such harmonic function, any level surface $H(x, y, z)=C$ is seen to be a cylindrical surface normal to the plane $x+y+z=0 . H(x, y, z)$ is thus equivalent, under a rigid motion carrying the complex plane into the plane $x+y+z=0$, to an ordinary 2dimensional harmonic function generated by an analytic function of the complex variable $x+i y$. (Received October 14, 1952.)

60t. C. N. Moore: On the infinitude of prime triplets. 
A question closely related to the infinitude of prime pairs is that of the infinitude of prime triplets, namely the infinite repetition of sequences of three primes whose successive differences are 2,4 , like $5,7,11$, or 4,2 , like $7,11,13$. The method which the author used for the discussion of the prime pair problem can be extended to this other problem by making suitable modifications. Consider three infinite columns of numbers formed by adding successive multiples of 30 to $7,11,13$. The multiples of 7 in the first column form an arithmetic progression with common difference 210 . The numbers in columns two or three opposite the terms of this progression form arithmetic progressions whose first terms and common differences are relatively prime. One then has formulas for the total number of primes in these progressions which are $\leqq$ a given number $x$. By considering also all composites in the first column which are multiples of $11,13, \cdots, p_{\lambda}$, where $p_{\lambda}$ is the greatest prime $\leqq x^{1 / 2}$, and also those which are multiples of the product of two or more of these primes, one can get an estimate of the number of primes in columns (2) or (3) which are opposite to composites in column (1). Similarly one can estimate the number of primes in column (3) which are opposite to composites in (2). The totality of primes in (3) $\leqq x^{1 / 2}$ is found to exceed the totality of primes opposite composites, as above determined. Thus the announced result is obtained. The infinite repetition of prime quadruplets and other similar prime groups can be discussed in analogous fashion. (Received October 14, 1952.)

\section{E. J. Pellicciaro: A study of critical sets of functions.}

A study of critical sets is made by use of their related complementary domains. $f(x)$ is a single-valued, continuous function of the $n$-real variables $x_{1}, \cdots, x_{n}$ throughout $R+C$, where $R$ is a bounded, connected, open subset of the $n$-dimensional Euclidean space and $C$ is its boundary. On $C, f(x)=K$, a constant, while at all points of $R, f(x)<K$. By a critical point of $f(x)$ is meant a point $p$ of $R$ such that there exists $\epsilon>0$ with the property that $[f(p+m E)-f(p)][f(p+n E)-f(p)] \geqq 0$ for all unit vectors $E$ and all scalars $m, n$ satisfying $0<|m|,|n|<\epsilon$. The critical set $M(H, p)$ that contains $p$ is defined to be that component of $H$, the set of all critical points of $f(x)$ on which $f(x)$ is constant, which contains $p$. With the aid of a few theorems concerning $M(H, p)$ and the complementary domains of $G[f=f(p)]$ with respect to $R$, a classification of critical sets is realized. This classification is exhaustive and mutually exclusive, dividing the collection of critical sets of $f(x)$ into five types, type 0 , hitherto not encountered, and types $1,2,3$, and 4, respectively comparable to the maximal, minimal, min-max, and flex types. (Received November 28, 1952.)

62. A. L. Shields: On additive properties of real numbers. Preliminary report.

Let $A$ and $B$ be two sets of real numbers. $A+B$ is the set of all numbers $a+b$, $a$ in $A, b$ in $B$. Then $m_{0}(A+B) \geqq m_{0}(A)+m_{0}(B)$ where $m_{0}$ is Lebesgue outer measure. Result true for inner measure. Proof consists in showing $A+B$ contains a translate of $A$ and a translate of $B$ which are almost disjoint. Let $R$ be reals, and $R_{1}$ the reals mod 1. Let $A, B$ be Borel subsets of $R_{1}$, and $m(A)+m(B) \leqq 1$. Then $m(A+B)$ $\geqq m(A)+m(B)$ where the addition in $A+B$ is mod 1 . Proof consists in reducing the problem to additive number theory by means of ergodic theory. $A$ is said to be rational (integral) basis for $R\left(R_{1}\right)$ if every $x$ in $R\left(R_{1}\right)$ may be represented as a finite linear combination of elements of $A$ with rational (integral) coefficients. Uniqueness of representation is not required. If $A$ is a Borel set and a rational basis for $R\left(R_{1}\right)$ then it is an integral basis. Let $A$ be an integral basis for $R_{1}$. It is said to be of finite order if 
there is an $N$ such that every $x$ in $R_{1}$ admits a representation in which the sum of the magnitudes of the coefficients doesn't exceed $N$. If $A$ is a Borel set and an additive basis for $R_{1}$, then it is of finite order. Finally, let $A$ have positive measure and let $F$ be a finite set. Then there exist numbers $r, s$ such that $r F+s \subset A$. (Received October 17, 1952.)

\section{G. N. Wollan: Euler methods of summability for double series.}

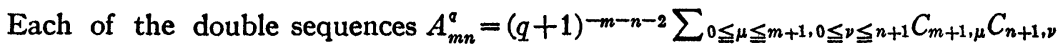
$q^{m+n+2-\mu-\nu} A_{\mu-1, \nu-1}$, where $A_{\mu \nu}=0$ whenever $\mu$ or $\nu$ is negative, and $\bar{A}_{m n}^{a}=(q+1)^{-m-n}$

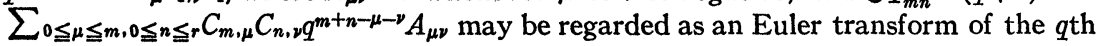
order of the double sequence $A_{m n}$. In this paper it is shown that for any $q \geqq 0$ if $\lim _{m, n \rightarrow \infty} A_{m n}^{a}=A$, then $\lim _{m, n \rightarrow \infty} A_{m n}^{a}=A$, but that the converse is not true. It is also

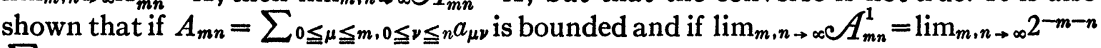

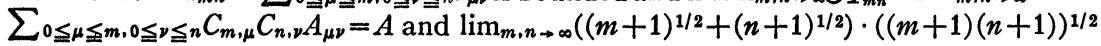
$\cdot a_{m n}=0$, then $\lim _{m, n \rightarrow \infty} A_{m n}=A$. (Received October 14, 1952.)

\section{Applied Mathematics}

\section{A. T. Hind, Jr.: On the convergence of approximate solutions of} the two-dimensional wave equation to the exact solution.

It is shown that approximate solutions of the two-dimensional wave equation can be obtained which converge to the exact solution for any mesh-ratio $r$ greater than zero, where $r(\Delta x)=\Delta t$ and $r(\Delta y)=\Delta t$. This is an extension of a paper on the onedimensional wave equation by Leutert and $O^{\prime} B$ rien in which they extend the values of the mesh-ratio $r$, for which approximate solutions converge to the exact solution, from $0<r \leqq 1$ to $0<r$. The convergence of approximate solutions of the two-dimensional wave equation is shown by the use of a lemma which treats all $0<r$ in one case instead of in three cases as required by the method of Leutert and O'Brien. As it is to be desired, this lemma contains theirs as a special case. (Received October 15, 1952.)

\section{W. R. Mann: Mean value methods in iteration.}

In order to use the Schauder fixpoint theorem to prove that a given problem has a solution, one must somehow associate with the problem a convex compact set in a Banach space and a continuous transformation which carries the set into itself. Assume that the fixpoint has been proved to be a solution of the original problem and assume furthermore that this solution has been proved to be unique. The problem then is: Given a convex compact set $E$ in a Banach space and a continuous transformation, $T$, carrying $E$ into itself and having a unique fixpoint, $x^{*}$, in $E$, construct a sequence of elements which converges to $x^{*}$. Assuming the ordinary interation process to fail, one considers the following modified iteration scheme. Taking $x_{1}$ to be an arbitrary point in $E$, we define $x_{n+1}=T\left(v_{n}\right)$ where $v_{n}=(1 / n) \sum_{k=1}^{n} x_{k}$. The following results are obtained. If either of the sequences $\left\{x_{n}\right\}$ and $\left\{v_{n}\right\}$ converge, then the other does and they have the common limit $x^{*}$. In the special case where $E$ is a line segment, $\left\{x_{n}\right\}$ always converges to $x^{*}$. In more general spaces properties of the sets of accumulation points of $\left\{v_{n}\right\}$ and $\left\{x_{n}\right\}$ are obtained. (Received October 14, 1952.)

\section{C. B. Smith: An infinite orthotropic plate containing a disk of a different orthotropic material.}

A large rectangular orthotropic plate containing a small circular disk of a different 
orthotropic material subjected to a uniform tension is discussed. The axes of elastic symmetry of the plate and the disk are taken to be parallel and, for simplicity, the uniform tension is assumed to act parallel to one of these common directions. The resulting stress distribution is derived from two stress functions, one stress function giving the stresses in the region exterior to the disk and the other giving the stresses interior to the disk. (Received October 14, 1952.)

\section{GEOMETRY}

\section{D. O. Ellis: $A$ modification of the parallelogram law character- ization of Hilbert spaces.}

Denote the "parallelogram law" which characterizes Hilbert spaces (real) among Banach spaces (real) by $(t)$. Using methods of vector lattice and metric lattice theory, the classical characterization ((1) is equivalent to (4) below) is extended to: Theorem. The following four conditions are pairwise equivalent for a Banach space (real) $B:$ : (1). $B$ is a Hilbert space. (2) There exists a partial ordering in $B$ so that $B$ is a Banach lattice and $(+)$ holds over the $B^{+}$defined by this ordering. (3) There exists a partial ordering of $B$ so that $B$ is a Banach lattice, the $B^{+}$defined by this ordering is a metric lattice under the norm $\nu(x)=\|x\|^{2}$, and $(\dagger)$ holds for pairs of comparable elements in this $B^{+}$. (4) ( () holds over $B$. It is also shown that any Hilbert space which is a Banach lattice (and any Hilbert space can be made one by the main theorem) is a UMB lattice and that if $B$ is a UMB lattice, the Banach space norm is strictly monotone increasing on $B^{+}$. (Received September 26, 1952.)

68t. Jack Levine: A canonical form for a conformally flat symmetric space.

It is shown that if a conformally flat space satisfies the conditions $R_{i j k m, p}=0$ (definition of a symmetric space of Cartan), then a coordinate system exists in which the quadratic form may be written as $d s^{2}=\left(1 / u^{2}\right) \sum e_{i}\left(d x^{i}\right)^{2}\left(e_{i}= \pm 1\right)$, where $u^{2}=a R^{2}$ $+L R+Q, R \equiv \sum_{i}^{n} e_{i}\left(x^{i}\right)^{2}, L \equiv \sum b_{i} e_{i} x^{i}, Q \equiv c_{i j} x^{i} x^{j}+2 d_{i} x^{i}+f$, with $a, b_{i}, c_{i j}, d_{i}$, and $f$ constants. This canonical form is obtained by the actual solution of the differential equations characterizing the above named space. (Received October 9, 1952.)

\section{TOPOLOGY}

\section{C. E. Capel: Inverse limit spaces. I. (Preliminary report.)}

The continuity axiom for a cohomology theory states that if $\left\{\left(X_{\lambda}, A_{\lambda}\right), \Pi_{\lambda \mu}, \Lambda\right\}$ is an inverse mapping system of compact pairs, then the pth cohomology group of the inverse limit is isomorphic, under a natural homomorphism, to the direct limit of the induced direct system $\left\{H^{p}\left(X_{\lambda}, A_{\lambda}\right), \Pi_{\lambda \mu}^{*}, \Lambda\right\}$. It is known that the continuity axiom implies the extension and reduction theorems. In the presence of the EilenbergSteenrod axioms, it is shown that the extension and reduction theorems imply the continuity axiom. The principal lemma is analogous to a fundamental lemma of $\mathrm{E}$. Spanier (Ann. of Math. vol. 49 (1948) pp. 407-427). (Received October 17, 1952.)

70. J. W. Ellis: Complementary spaces of cartesian products of groups and linear spaces.

Let $X$ be called an $S$-space if $X$ is a topological group and $S$ a commutative semigroup whose members act as continuous functions on $X$ and leave the identity fixed. If $Z$ is a second $S$-space, the $Z$-complement of $X$ is the group of all continuous homo- 
morphisms on $X$ to $Z$ which "commute with the members of $S$." If, for each $\alpha, X_{\alpha}$ is an $S$-space, it is true, under certain conditions on $Z$, that the $Z$-complement of $P X_{\alpha}$ is the weak product of the $Z$-complements of the $X_{\alpha}$. Similarly, the $Z$-complement of the weak product of the $X_{\alpha}$, when this space has a suitable topology, is isomorphic to the full cartesian product of the $Z$-complements. The conditions on $Z$ are satisfied by the reals as a real-space, and by the reals modulo one as a space over the integers; this theorem is then shown to include Kaplan's result on the character group of a product of groups, and Katertov's theorem on the adjoint of a product of linear spaces. Other applications generalize results by Dixmier, by Šmulian, and by Hewitt and Zuckermann. (Received October 14, 1952.)

\section{Mary E. Estill: An effect of completeness.}

The purpose of this paper is to answer two questions raised by F. B. Jones, the first in a paper entitled Connected and disconnected plane sets and the functional equation $f(x)+f(y)=f(x+y)$ which appears in the February, 1942 issue of the Bulletin of the American Mathematical Society, and the second in a paper entitled Certain consequences of the Jordan curve theorem which appears in the July, 1941 issue of the American Journal of Mathematics. It is shown that the following theorem settles both questions and its proof is given. There is a connected, linear, complete Moore space in which there exists a totally disconnected domain. (Received October 14, 1952.)

\section{W. L. Gordon: On the coefficient group in cohomology.}

Cohomology groups of the Alexander-Kolmogoroff type may be defined using only $p$-cocycles which locally are finite-valued. For fully normal Hausdorff (=paracompact) spaces, the groups so defined are naturally isomorphic with the usual AlexanderKolmogoroff groups and hence with the Cech groups based on arbitrary coverings. In the locally-compact case it is proven that a cocycle with compact support has a finitely valued cocycle in the same cohomology class. For compact Hausdorff-closed pairs $X, A$ the following results are obtained: If the abelian coefficient group $G$ is the direct sum of groups $G_{\alpha}$, then the pth cohomology group $H^{p}(X, A ; G)$ of the pair $(X, A)$ with coefficients in $G$ naturally decomposes as the direct sum of the groups $H^{p}\left(X, A ; G_{\alpha}\right)$. An analogous result is obtained in case $G$ is the direct limit of a direct system of groups, reducing the problem of the universal coefficient group to a problem involving only finitely-generated coefficient groups. Applications include an algebraic proof that the use of arbitrary coefficient groups in a cohomological definition of dimension will yield a numerical invariant that cannot exceed the (usual) dimension. The classical equality of the $p$ th Betti number of a finite polyhedron with the dimension of the $p$ th homology group over the rational field is given a natural extension to compact spaces. (Received October 15, 1952.)

\section{3t. J. S. Griffin, Jr.: A note on transfinite sequences.}

A transfinite sequence is defined to be a net (for definition see J. L. Kelley, Duke Math. J. vol. 17, pp. 277-285) whose domain is a limit ordinal. If $X$ is a topological space and $A \subset X$, then (1) $x$ is in the closure of $A$ iff there is a transfinite sequence in $A$ clustering at $x$; (2) a function $f$ on $X$ is continuous iff $f$ carries cluster points of transfinite sequences in $X$ into cluster points of their images; (3) $X$ is compact iff each transfinite sequence in $X$ has a cluster point; (4) $X$ is Frechet-compact iff each (ordinary) sequence in $X$ has a cluster point. There may be constructed a topological space with a subset $A$ which has an accumulation point $x$ but such that no transfinite 
sequence in $A$ converges to $x$ (although there are those in $A$ which cluster at $x$ ). (Received October 20, 1952.)

\section{J. R. Isbell: Notes on homogeneous spaces.}

All microhomogeneous linear continua are homogeneous, and either Birkhoff linear homogeneous continua or uncountable unions of such continua. With reference to van Dantzig, Über das topologische homogene Kontinua, Fund. Math. vol. 16 (1930) pp. 102-125: the classification of homogeneities and the product theorem are extended and a problem is solved: all locally Euclidean manifolds are two-point homogeneous but one is not involutory homogeneous. (Received November 26,1952.)

\section{5t. J. R. Isbell: Unicoherence of meets and joins.}

The union of a chain of locally connected open sets each component of each of which is unicoherent is disconnected or unicoherent. The intersection of a chain of locally connected compact Hausdorff sets each component of each of which is unicoherent is disconnected or unicoherent. The intersection theorem generalizes Borsuk's corresponding result for Peano continua (Fund. Math. vol. 17 (1931) p. 208); it is proved by use of the lemma: unicoherence of normal connected locally connected spaces is equivalent to the property that if $A$ and $B$ are connected open sets covering the space, $\overrightarrow{A \cap B}$ is connected. (Received September 15, 1952.)

\section{R. J. Koch: A lemma on mobs.}

Following A. D. Wallace, we define a mob to be a Hausdorff space which admits a continuous, associative multiplication. The purpose of this note is to exhibit the following lemma: Let $X$ be a mob, and $A$ a compact subset of $X$, let $\left\{p_{\alpha}\right\}$ be a net (generalized sequence) in $X$, and $p_{0}$ any cluster point of $\left\{p_{\alpha}\right\}$; suppose $A p_{\beta} \subset A p_{\gamma}$ if $\beta>\gamma$. Then $\bigcap_{\alpha} A p_{\alpha}=A p_{0}$. Wallace has shown that every compact mob contains at least one idempotent. It follows as a consequence of this result with the present lemma that if $X$ is a compact mob and $a \in X$, then there is an idempotent $e$ such that $\bigcap_{n} X a^{n}=X e$. (Received November 24, 1952.)

\section{L. F. McAuley: On the aposyndetic decomposition of continua.}

Suppose that $M$ is a continuum and that $p$ is a point of $M$. Let $M(p)$ denote the set of all points $x$ of $M$ such that there does not exist an uncountable collection $G(p, x)$ of mutually exclusive closed subsets of $M$ such that (1) no element of $G(p, x)$ separates an element of $G(p, x)$ in $M$ and (2) each element $g$ of $G(p, x)$ separates $p$ from $x$ in $M$. An example is given in the plane of a compact continuum $M$ which contains a point $p$ such that $M(p)$ is not connected. With the aid of several lemmas, the following theorem is proved. If $M$ is a compact metric continuum and $H$ is the collection of all continua $C$ such that, for some point $p$ of $M, C$ is a component of $M(p)$, then $H$ is an upper semi-continuous collection of mutually exclusive continua filling up $M$; and furthermore, with respect to its elements as points, $H$ is a compact aposyndetic metric continuum. An example is given in the plane to show that $H$ is not necessarily connected im kleinen. (Received October 14, 1952.)

\section{W. L. Strother: Multi-homotopy.}

Definitions are given for (1) homotopy of multi-valued functions, (2) multiretracts, and (3) multi-homotopy groups. Tietze's extension theorem is generalized and a theorem is proved which enables one to generalize numerous theorems on homot- 
opy groups to multi-homotopy groups. (Received October 14,1952.)

\section{P. M. Swingle: $A$ note on $n$-point connected sets.}

A connexe $C$ is $n$-point connected if there does not exist a subset of power $n$ whose omission disconnects $C$ (Bulletin, 1931, p. 254). A connexe $C$ will be said to be $n$ densely connected if there exist $n$, but not a greater cardinal number, of mutually exclusive subconnexes, each dense in $C$. The main problem of this note deals with the existence of $n$-densely connected subsets, and related subconnexes, in an (aleph-null)point connected set. (Received October 14, 1952.)

\section{M. J. Walsh: The paracompactness of the CW-complex.}

J. H. C. Whitehead (Bull. Amer. Math. Soc. vol. 55 (1949) pp. 213-245) has introduced the concepts of the generalized cell complex and the weak topology in complexes. It is shown that a closure finite generalized cell complex with the weak topology (CW-complex) is paracompact. An equivalent condition for the paracompactness of a space is also given. (Received October 14, 1952.)

\section{H. C. Wang: Compact manifolds with homogeneous complex structure.}

All manifolds here are assumed to be simply-connected and closed. Very few examples are known of a manifold which either (a) admits infinitely many inequivalent complex structures, or (b) admits a nonalgebraic complex structure. Hizerbruch exhibited two spaces with property (a), and later Calabi-Eckmann showed that $S^{2 p+1}$ $\times S^{2 q^{+1}}$ has both properties (a) and (b). In this paper, all homogeneous complex manifolds are determined. As consequences, we find that nonalgebraic complex manifolds are not so limited and most of them in the homogeneous case have property (a). The main results are as follows: (I) Each homogeneous complex manifold is homeomorphic with a fibre decomposition space, with torus as fibre, of a product of certain spaces, called elementary spaces. All elementary spaces are determined. Among them are, in particular, the Stiefel manifolds $V_{n, 2 k}$, the complex Stiefel manifolds $W_{n, k}$ (odd spheres are included as special cases), and the space of compact simple Lie groups. (II) The product of elementary spaces is nonalgebraic, and has a homogeneous complex structure when its dimension is even. (III) The number of differentiably inequivalent homogeneous complex structures over a manifold is either noncountable or finite according as the Euler characteristic vanishes or not. (Received October 14,1952.)

W. M. Whyburn, Associate Secretary 